\title{
Stable states of a relativistic bilocal stochastic oscillator: a new quark-lepton model
}

\author{
N Cufaro Petroni $\dagger$, Z Marićł, Dj Živanović§ and J P Vigier|| \\ † Istituto di Fisica dell' Università Bari and Istituto Nazionale di Fisica Nucleare-Sezione \\ Bari, Bari, Italy \\ $\ddagger$ Institue of Physics, Belgrade, Yugoslavia \\ $\S$ Department of Physics, University of Belgrade, and Laboratory for Theoretical Physics, \\ Institute 'Boris Kidric', Belgrade, Yugoslavia \\ \| Equipe de Recherche Associée au CNRS no 533, Institut Henri Poincaré, 11 rue Pierre et \\ Marie Curie, 75231 Paris Cedex 05, France
}

Received 24 April 1980 , in final form 28 July 1980

\begin{abstract}
We analyse a stochastic linearised extension of the Yukawa-Takabayasi-Feynman bilocal oscillator model and show that: (a) the external Poincaré group P commutes with an internal extension of the Lorentz group, i.e. $\mathrm{U}(1) \otimes \mathrm{SO}(6,2) ;$ (b) the corresponding internal fundamental spinor representation of the associated $D_{4}$ algebra yields eight quarks and eight leptons, which correspond to heuristic proposals of Nambu and Salam.
\end{abstract}

All recent attempts to classify elementary quarks and leptons start from the heuristic introduction of assumed gauge groups and Yang-Mills interactions, completed with ad hoc Higgs multiplets. These theories, illustrated by Salam (1968) and Weinberg (1967), justifiably claim important success, but leave open the question of the origin of the new internal 'charges', as well as the cause of relations of the type $Q=T+Y / 2$ which connect quantum numbers associated with couplings of a different physical nature.

The aim of the present paper is to revive and develop an alternative line of research started by Yukawa $(1950 a, b, 1953,1956)$. In this type of model, particles are extended time-like hypertubes in space-time which can be represented, in the first approximation, by bilocal structures that yield internal quantised states corresponding to quarks and leptons. This model has been studied in quantum form by Feynman et al (1971) and essentially developed by Takabayasi (1965a, b, 1968, 1979).

Until now, various attempts along this line have failed to produce satisfactory results (Katayama 1963, de Broglie et al 1963). The new step taken here is to introduce (in the trail of recent developments in the stochastic interpretation of quantum mechanics (Vigier 1979, Cufaro Petroni and Vigier 1979)) the following idea: to add to the space-time coordinates $x_{1}$ and $x_{2}$ of the two points, bound together by a relativistic harmonic oscillator potential, supplementary stochastic motions $\delta x_{1}$ and $\delta x_{2}$, which reflect the action of an isotropic constant thermostat. They, as well as their corresponding momenta $\delta p_{1}$ and $\delta p_{2}$, satisfy the relations $\left\langle\delta x_{1,2}\right\rangle=\left\langle\delta p_{1,2}\right\rangle=0$, where $\langle\ldots\rangle$ represents averages taken on four-dimensional volume elements in configuration space. 
Before we do this, let us briefly revisit Feynman's presentation of Yukawa's model, in order to discuss its linearisation and the connection between external Poincaré and internal homogeneous Lorentz groups of motion. Feynman's equation for the twobody bound state is (Feynman et al 1971)

$$
\left[2\left(\square_{x_{1}}+\square_{x_{2}}\right)-\left(\omega^{2} / 16\right)\left(x_{1}-x_{2}\right)^{2}+m_{0}^{2}\right] \phi\left(x_{1}, x_{2}\right)=0 .
$$

By introducing the centre-of-mass and relative variables (Kim et al 1978) $Q=\frac{1}{2}\left(x_{1}+x_{2}\right)$ and $q=\left(\frac{1}{2} \sqrt{2}\right)\left(x_{1}-x_{2}\right)$, it can be written in the form (for $\omega=1$ )

$$
\left[\square_{Q}+m_{0}^{2}+\frac{1}{2}\left(\square_{q}-q^{2}\right)\right] \phi(Q, q)=0
$$

separable in the $Q$ and $q$ variables; writing $\phi(Q, q)=\psi_{\mathrm{E}}(Q) \psi_{\mathrm{I}}(q)$ one obtains the equations

$$
\left(\square Q+m_{0}^{2}+\epsilon\right) \psi_{\mathrm{E}}(Q)=0
$$

and

$$
\frac{1}{2}\left(\square_{q}-q^{2}\right) \psi_{\mathrm{I}}(q)=\epsilon \psi_{\mathrm{I}}(q)
$$

Equation (3) describes the external motion of the bilocal system: it is a Klein-Gordon equation, and its solution of the form $\psi_{E}(Q)=\exp (-i P Q)$ (with $P^{2}=M^{2}=m_{0}^{2}+\epsilon$ ) corresponds to the free motion of the system. The external wavefunction $\psi_{\mathrm{E}}(Q)$ transforms under the usual external Poincaré group $\mathrm{P}=\mathrm{T} \otimes \mathrm{SO}(3,1)$, since under a translation $x_{1,2} \rightarrow x_{1,2}+a$ we have $Q \rightarrow Q+a$. The case of the internal wavefunction $\psi_{1}(q)$ is different, as the relative coordinate $q$ remains invariant under translation. Accordingly, it transforms under homogeneous Lorentz transformation. For the scalar case, considered in Feynman et al (1971) there is no problem. The problem arises when we linearise equations (3) and (4) into

$$
(\gamma \hat{P}-M) \psi_{E, \alpha}(Q)=0
$$

and

$$
(\gamma \hat{p}+\gamma q+\mathrm{i} \sqrt{2} \epsilon) \psi_{\mathrm{I}, \alpha}(q)=0
$$

to obtain external and internal spinors, $\psi_{\mathrm{E}, \alpha}(Q)$ and $\psi_{\mathrm{I}, \alpha}(q)$. Here $\hat{P}$ and $\hat{p}$ correspond to the operators $\mathrm{i} \partial_{Q}$ and $\mathrm{i} \partial_{q}$, respectively. As is known, (Takabayasi 1979) it is not possible to linearise (4), unless we impose the supplementary condition $q \hat{p} \psi_{\mathrm{I}, \alpha}(q)=0$. If this is done, then we can introduce commuting external and internal $\mathrm{SO}(3,1)$ transformations by utilising Chevalley's (Chevalley 1946, Halbwachs and Souriau 1964) left and right translations $\mathrm{SO}(3,1)_{\mathrm{L}, \mathrm{R}}$, of the homogeneous Lorentz group $\mathrm{SO}(3,1)$. This bilateral group BilSO $(3,1)$ is equal to $\mathrm{SO}(3,1)_{\mathrm{L}} \otimes \mathrm{SO}(3,1)_{\mathrm{R}}=(\mathrm{SO}(3,1) \otimes \mathrm{SO}(3,1)) / C$, where $C$ is the centre of the whole group. Such commuting left and right Lorentz translations (having the same Casimir operators) have already been used by de Broglie et al (1963) in their rotator particle model. Now, $\operatorname{SO}(3,1)_{\mathrm{L}}$ and $\mathrm{SO}(3,1)_{\mathrm{R}}$ (which can be represented by three-dimensional complex rotations) correspond to external and internal transformations respectively, i.e. to angular momentum projections on Einstein tetrads associated with the observer and the particle. Moreover, one sees that for $\Omega=0$ the model reduces to the rotator model of de Broglie et al (1963).

Now we introduce our stochastic motion, as was done by Guéret et al (1979) (denoted I hereafter). The variable $Q$ becomes $Q+\delta Q$, but nothing is changed in the average motion of $Q$, since the internal temperature does not modify the centre-ofmass motion. The relative variable $q$ goes into $q+\delta q$, but then equations (4) and $(4 a)$ 
transform according to the $(6+2)$-dimensional group of motion, introduced in I to describe the motion of a relativistic oscillator embedded in a random stochastic thermostat.

To include the stochastic behaviour in the motion described by equation (4), which evidently stems from the Hamiltonian

$$
H_{\mathrm{I}}=(1 / 2 m)\left(p^{2}+\omega^{2} q^{2}\right)
$$

we note that the stochastic contributions $\delta q$ and $\delta p$ behave like new independent variables, so that we can consider the total set of variables as describing two independent points (i.e. $q$ and $\delta q$ ) in a $(6+2)$-dimensional configuration space. Of course, if one assumes an isotropic constant thermostat, we have $\langle q\rangle=q$ and $\langle p\rangle=p$ along with $\langle\delta q\rangle=\langle\delta p\rangle=0$, where the \langle\rangle represents averages taken on four-dimensional volume elements in configuration space. As is known, any motion in our new 16-dimensional phase space implies an assumption on the connection of the sets $(q, p)$ and $(\delta q, \delta p)$. If we limit ourselves to the descriptions of small (regular plus random) motions at the bottom of an arbitrary potential well, (i.e. $\Gamma_{0}$ harmonic oscillations) we can generalise $H_{1}$ (in equation $(5 a)$ ) into

$$
H_{\mathrm{I}}^{\prime}=(1 / 2 m)\left[(p+\delta p)(p+\delta p)+\omega^{2}(q+\delta q)(q+\delta q)\right]
$$

which yields the corresponding Liouville equation. This yields an average motion described by the average Hamiltonian $\left\langle H_{\mathrm{I}}^{\prime}\right\rangle$ which can be calculated. Indeed since we have $\langle A B\rangle=\langle A\rangle\langle B\rangle$ for any pair of independent variables $A, B$ (with $A \neq B$ ) in phase space we obtain (with $\langle q\rangle=q$ and $\langle p\rangle=p$ )

$$
H_{\mathrm{I}}=\left\langle H_{\mathrm{I}}^{\prime}\right\rangle=(1 / 2 m)\left[p^{2}+\delta p^{2}+\omega^{2}\left(q^{2}+\delta q^{2}\right)\right]
$$

which describes motions in our $2(6+2)$-dimensional phase space and has been analysed from Cartan's point of view by Guéret et al (1973), denoted II hereafter.

The Hamiltonian (6) and its linearised form given in II are invariant under the symplectic group $\mathrm{Sp}(12,4)$ and admit as the general symmetry group $\mathrm{U}(6,2) \supset$ $\mathrm{SU}(1,1) \otimes \mathrm{SO}(6,2)$, where $\mathrm{SO}(6,2)$ now contains $\mathrm{SO}(3,1)_{\mathrm{R}}$, as its subgroup which acts on the two distinct spinor representations, 8 and $\overline{8}$ (along with a third, vector representation $8^{\prime}$ ), interchangable under the discrete automorphisms of the corresponding $\mathrm{D}_{4}$ Lie algebra analysed by Cartan (1938).

We note here that the introduction of internal stochastic motions (represented by $\delta q$ ) is mathematically equivalent to doubling the number of space-time coordinates, i.e. to move into an extended configuration space. In particular, the new internal time coordinate represents the relative time projection of the random part of the motion on the particle's rest mass frame. This solves the age-old problem of interpreting the new internal times which necessarily appear in bilocal or extended particle models.

If one then further transforms $\mathrm{H}_{\mathrm{I}}$ to a Feynmann-Gell'Mann type of equation, i.e. to $H_{\mathrm{I}}^{\prime}=(1 / 2 m) P^{2}$, which can be linearised into $H_{\mathrm{I}}^{\prime}=(1 / 2 m)(\Gamma p)$, with the help of the 16-dimensional matrices $\Gamma$ calculated in $\mathrm{I}$, we can assume that the wavefunctions $\psi_{\mathrm{E}}$ and $\psi_{\mathrm{I}}$ are simultaneously spinors, or vectors, and classify all the various fermionic particles into the two spinor families $\psi_{\mathrm{I}}^{\prime}(8)$ and $\psi_{\mathrm{I}}^{\prime \prime}(\overline{8})$, the corresponding antiparticles belonging to opposite values of $\mathrm{U}(1)$ in $\mathrm{G}=\mathrm{U}(1) \otimes \mathrm{SO}(6,2)$, which leaves invariant $H_{\mathrm{I}}^{\prime}$ and $H_{\mathrm{I}}^{\prime}$. The Weyl-Cartan algebra of $\mathrm{SO}(6,2)$ explicitly calculated by Guéret et al in II and Vigier (1976) (denoted III) yields (along with the Casimir invariant operators) four diagonal commuting operators $H_{i}(i=1,2,3,4)$ and 24 'raising' and 'lowering' operators $E_{\alpha}$ and $E_{-\alpha}$ with $\left[E_{\alpha}, E_{-\alpha}\right]=\frac{1}{8} \alpha_{i} H_{i}$, where $\alpha_{i}$ denotes a root and $H_{1}=M_{12}$, 
$H_{2}=M_{34}, H_{3}=M_{56}$ and $H_{4}=M_{78}$. Moreover, $x_{1}^{2}+\ldots+x_{6}^{2}-x_{7}^{2}-x_{8}^{2}=$ constant in $E_{6,2}$. We thus obtain for $J=1 / 2^{+}$(external spinors) eight states of the same 'colour' denoted yellow (i.e. y), corresponding to the finite non-unitary representation (8) (see table 1) with four quarks and four leptons belonging respectively to the representations (4) and $(\overline{4})$ of the subgroup $\mathrm{SO}(6)=\mathrm{SU}(4)$ with opposite $H_{4}$ values, each state is characterised by the eigenvalue $H_{0}$ of $\mathrm{U}(1)$ and one spinor component of the ket $E_{\alpha}=$ $\left.\mid H_{1}, H_{2}, H_{3}, H_{4}\right)$ determined by Cartan (1938). These $H$-values are given in the first five columns of table 1 ; the last columns give, as in III, the usual quantum numbers: $T_{3}=\left(H_{1}-H_{2}\right) / 2, \quad Y=\left(\frac{1}{3}\right)\left(H_{1}+H_{2}-2 H_{3}\right), Z=-\left(\frac{1}{2}\right)\left(H_{1}+H_{2}+H_{3}\right), Q=T_{3}+(Y / 2)-$ $(2 Z / 3)-\left(H_{4} / 2\right)=H_{1}+H_{4}$, along with $S=H_{1}+H_{2}$ and $C$ (charm $)=\left(3 H_{4} / 2\right)-Z$ which we have determined (following Yukawa's (1956) and Okubo's (1978) suggestions, that corresponds to Yang-Mills gauge fields) from a choice of compact subgroups of $\mathrm{G}_{\mathrm{I}}$ preserved in typical superpositions (combinations) of our basic oscillating states.

Table 1.

\begin{tabular}{|c|c|c|c|c|c|c|c|c|c|c|c|c|c|c|}
\hline & $\mathrm{H}_{0}$ & $\mathrm{H}_{1}$ & $\mathrm{H}_{2}$ & $\mathrm{H}_{3}$ & $\mathrm{H}_{4}$ & $T_{3}$ & $\mathrm{Y}$ & z & $S_{w}$ & $C$ & $Q$ & Particle & $\mathrm{SU}(3)$ & SU(2) \\
\hline$\xi_{123}$ & -1 & $-\frac{1}{2}$ & $-\frac{1}{2}$ & $-\frac{1}{2}$ & $+\frac{1}{2}$ & 0 & 0 & $+\frac{3}{4}$ & -1 & 0 & 0 & $c^{y}$ & singlet & \multirow{4}{*}{$\begin{array}{l}\text { doublet } \\
H_{3}=+\frac{1}{2}\end{array}$} \\
\hline$-\xi_{3}$ & -1 & $+\frac{1}{2}$ & $+\frac{1}{2}$ & $-\frac{1}{2}$ & $+\frac{1}{2}$ & 0 & $+\frac{2}{3}$ & $-\frac{1}{4}$ & +1 & +1 & +1 & $s^{y}$ & \multirow{3}{*}{ triplet } & \\
\hline$-\xi_{1}$ & -1 & $+\frac{1}{2}$ & $-\frac{1}{2}$ & $+\frac{1}{2}$ & $+\frac{1}{2}$ & $+\frac{1}{2}$ & $-\frac{1}{3}$ & $-\frac{1}{4}$ & 0 & +1 & +1 & $u^{y}$ & & \\
\hline$-\xi_{2}$ & -1 & $-\frac{1}{2}$ & $+\frac{1}{2}$ & $+\frac{1}{2}$ & $+\frac{1}{2}$ & $-\frac{1}{2}$ & $-\frac{1}{3}$ & $-\frac{1}{4}$ & 0 & +1 & 0 & $d^{y}$ & & \\
\hline$\xi_{4}$ & -1 & $+\frac{1}{2}$ & $+\frac{1}{2}$ & $+\frac{1}{2}$ & $-\frac{1}{2}$ & 0 & 0 & $-\frac{3}{4}$ & +1 & 0 & 0 & $\nu_{\tau}$ & singlet & \multirow{2}{*}{$\begin{array}{l}\text { doublet } \\
H_{3}=+\frac{1}{2}\end{array}$} \\
\hline$\xi_{124}$ & -1 & $-\frac{1}{2}$ & $-\frac{1}{2}$ & $+\frac{1}{2}$ & $-\frac{1}{2}$ & 0 & $-\frac{2}{3}$ & $+\frac{1}{4}$ & -1 & -1 & -1 & $\tau^{-}$ & \multirow{3}{*}{ triplet } & \\
\hline$\xi_{234}$ & -1 & $-\frac{1}{2}$ & $+\frac{1}{2}$ & $-\frac{1}{2}$ & $-\frac{1}{2}$ & $-\frac{1}{2}$ & $+\frac{1}{3}$ & $+\frac{1}{4}$ & 0 & -1 & -1 & $e^{-}$ & & \multirow[t]{2}{*}{$\begin{array}{l}\text { doublet } \\
H_{3}=-\frac{1}{2}\end{array}$} \\
\hline$\xi_{314}$ & -1 & $+\frac{1}{2}$ & $-\frac{1}{2}$ & $-\frac{1}{2}$ & $-\frac{1}{2}$ & $+\frac{1}{2}$ & $+\frac{1}{3}$ & $+\frac{1}{4}$ & 0 & -1 & 0 & $\nu_{e}$ & & \\
\hline
\end{tabular}

This choice of internal $H_{i}$ combinations to label the quantum numbers results in a unique way from our dynamical model for two reasons. The first is that the corresponding Yang-Mills fields must be associated with particular compact subgroups embedded in our general non-compact dynamical group. The second is that these subgroups must yield the SU(2) CSU(3) CSU(4) embedding which gives the correct generalisation of the Gell'Mann-Okubo formulae. Moreover, following Cartan, the choice of the $H_{i}$ to label the spinor components cannot be avoided. Indeed the $\mathrm{SO}(6,2)$ 28 generators contains 16 simultaneously diagonalisable commuting operators, out of which 12 are Casimir operators whose eigenvalues label the representations and four (i.e. the $H_{i}$ 's) differentiate the spinor (vector) components.

Of course, the corresponding antiparticles will have $J=1 / 2^{-}$and the opposite $H_{i}$-values, since they correspond to internal mirror motions in our scheme (Flato $\mathrm{et} \mathrm{al}$ 1965). Table 1 yields a unique combination of Salam's $(1974,1976) F_{e}$-type fermions with four yellow quarks $\mathrm{q}^{\mathrm{y}}$ and four leptons $\mathrm{l}^{\mathrm{y}}$. Curiously, these quarks are just the 'Yukawon' first proposed by Yukawa and discussed in the literature by de Broglie et al 
(1963). They can be mapped on Salam's (1974) and Pati and Salam's (1973, 1974a, b, 1975 ) integer quark classification.

Table 2 with $j=1 / 2^{+}$yields the (blue) $\overline{8}$ octet of particles (the antiparticles being obtained as for 8 ) which corresponds to $\mathrm{F}_{\mu}$-type fermions. The corresponding $\mathrm{SU}(3)$ quark triplet corresponds to Sakata's well known model.

The last fundamental octet of (coloured) gluon vector particles (table 3 ) splits into a SU(4) sextet (i.e. two SU(3) triplets) and two SU(4) singlets which ensure (II) quarklepton transition from 8 to $\overline{8}$ and vice versa. It has $J=1^{+}$. This also maps on Salam's (1974) Pati and Salam's $(1975,1976)$ and Pati et al's (1976) proposals.

We now mention some consequences of our model.

A. The model evidently contains the essential part of the strong-interaction results predicted by Nambu (Han and Nambu 1965), Pati and Salam in their integer-charged

Table 2.

\begin{tabular}{|c|c|c|c|c|c|c|c|c|c|c|c|c|c|c|}
\hline & $H_{0}$ & $H_{1}$ & $H_{2}$ & $\mathrm{H}_{3}$ & $H_{4}$ & $T_{3}$ & $Y$ & $z$ & $S_{\mathrm{w}}$ & $C$ & $Q$ & Particle & $\mathrm{SU}(3)$ & $\mathrm{SU}(2)$ \\
\hline$\xi_{0}$ & -1 & $+\frac{1}{2}$ & $+\frac{1}{2}$ & $+\frac{1}{2}$ & $+\frac{1}{2}$ & 0 & 0 & $-\frac{3}{4}$ & +1 & $+\frac{3}{2}$ & +1 & $c^{b}$ & singlet & \multirow{2}{*}{$\begin{array}{l}\text { doublet } \\
H_{3}=+\frac{1}{2}\end{array}$} \\
\hline$\xi_{12}$ & -1 & $-\frac{1}{2}$ & $-\frac{1}{2}$ & $+\frac{1}{2}$ & $+\frac{1}{2}$ & 0 & $-\frac{2}{3}$ & $+\frac{1}{4}$ & -1 & $+\frac{1}{2}$ & 0 & $s^{b}$ & \multirow{3}{*}{ triplet } & \\
\hline$\xi_{23}$ & -1 & $-\frac{1}{2}$ & $+\frac{1}{2}$ & $-\frac{1}{2}$ & $+\frac{1}{2}$ & $-\frac{1}{2}$ & $+\frac{1}{3}$ & $+\frac{1}{4}$ & 0 & $+\frac{1}{2}$ & 0 & $\mathrm{~d}^{b}$ & & \multirow[t]{2}{*}{$\begin{array}{l}\text { doublet } \\
H_{3}=-\frac{1}{2}\end{array}$} \\
\hline$\xi_{34}$ & -1 & $+\frac{1}{2}$ & $-\frac{1}{2}$ & $-\frac{1}{2}$ & $+\frac{1}{2}$ & $+\frac{1}{2}$ & $+\frac{1}{3}$ & $+\frac{1}{4}$ & 0 & $+\frac{1}{2}$ & +1 & $\mathrm{u}^{b}$ & & \\
\hline$-\xi_{1234}$ & -1 & $-\frac{1}{2}$ & $-\frac{1}{2}$ & $-\frac{1}{2}$ & $-\frac{1}{2}$ & 0 & 0 & $+\frac{3}{4}$ & -1 & $-\frac{3}{2}$ & -1 & $\mathbf{M}^{-}$ & nglet & \multirow{2}{*}{$\begin{array}{l}\text { doublet } \\
H_{3}=-\frac{1}{2}\end{array}$} \\
\hline$\xi_{34}$ & -1 & $+\frac{1}{2}$ & $+\frac{1}{2}$ & $-\frac{1}{2}$ & $-\frac{1}{2}$ & 0 & $+\frac{2}{3}$ & $-\frac{1}{4}$ & +1 & $-\frac{1}{2}$ & 0 & $\mathrm{M}^{e}$ & \multirow{3}{*}{ triplet } & \\
\hline$\xi_{14}$ & -1 & $+\frac{1}{2}$ & $-\frac{1}{2}$ & $+\frac{1}{2}$ & $-\frac{1}{2}$ & $+\frac{1}{2}$ & $-\frac{1}{3}$ & $-\frac{1}{4}$ & 0 & $-\frac{1}{2}$ & 0 & $\nu_{\mu}$ & & \multirow[t]{2}{*}{$\begin{array}{l}\text { doublet } \\
H_{3}=+\end{array}$} \\
\hline$\xi_{24}$ & -1 & $-\frac{1}{2}$ & $+\frac{1}{2}$ & $+\frac{1}{2}$ & $-\frac{1}{2}$ & $-\frac{1}{2}$ & $-\frac{1}{3}$ & $-\frac{1}{4}$ & 0 & $-\frac{1}{2}$ & -1 & $\mu^{-}$ & & \\
\hline
\end{tabular}

Table 3.

\begin{tabular}{lccccccccccccc}
\hline & $H_{0}$ & $H_{1}$ & $H_{2}$ & $H_{3}$ & $H_{4}$ & $T_{3}$ & $Y$ & $Z$ & $S_{\mathrm{w}}$ & $C$ & $Q$ & $\mathrm{SU}(3)$ & $\mathrm{SU}(4)$ \\
\hline$x^{4}$ & 0 & 0 & 0 & 0 & +1 & 0 & 0 & 0 & 0 & $+\frac{3}{2}$ & +1 & singlet & singlet \\
$x^{1}$ & 0 & +1 & 0 & 0 & 0 & $+\frac{1}{2}$ & $+\frac{1}{3}$ & $-\frac{1}{2}$ & +1 & $+\frac{1}{2}$ & +1 & & \\
$x^{2}$ & 0 & 0 & +1 & 0 & 0 & $-\frac{1}{2}$ & $+\frac{1}{3}$ & $-\frac{1}{2}$ & +1 & $+\frac{1}{2}$ & 0 & triplet & \\
$x^{3}$ & 0 & 0 & 0 & +1 & 0 & 0 & $-\frac{2}{3}$ & $-\frac{1}{2}$ & 0 & $+\frac{1}{2}$ & 0 & & \\
\hline$x^{3^{\prime}}$ & 0 & 0 & 0 & -1 & 0 & 0 & $+\frac{2}{3}$ & $+\frac{1}{2}$ & 0 & $-\frac{1}{2}$ & 0 & & \\
$x^{2}$ & 0 & 0 & -1 & 0 & 0 & $+\frac{1}{2}$ & $-\frac{1}{3}$ & $+\frac{1}{2}$ & -1 & $-\frac{1}{2}$ & 0 & triplet & \\
$x^{1^{\prime}}$ & 0 & -1 & 0 & 0 & 0 & $-\frac{1}{2}$ & $-\frac{1}{3}$ & $+\frac{1}{2}$ & -1 & $-\frac{1}{2}$ & -1 & & \\
$x^{4}$ & 0 & 0 & 0 & 0 & -1 & 0 & 0 & 0 & 0 & $-\frac{3}{2}$ & -1 & singlet & singlet \\
\hline
\end{tabular}


quark model. Indeed, if we assume that strong interactions preserve $C$ (i.e. $\mathrm{SU}(3)$ ) and $H_{0}$ (fermion) numbers, we see that boson multiplets are built with coloured $\mathrm{q} \overline{\mathrm{q}}$ combinations of the SU(3) triplets contained in $\overline{8}$ and 8: the corresponding $F_{e}$ and $F_{\mu}$ hadronic multiplets resulting from their multiplication by $\mathrm{SU}(3)$ singlets, i.e. $\mathrm{F}_{\mathrm{e}}\left(\mathrm{F}_{\mu}\right)=$ $\mathrm{q}(\mathrm{q} \overline{\mathrm{q}})$. The $\mathrm{SU}(3)$ gauge group contains the usual $J=1^{-} \mathrm{SU}(3) \mathrm{q} \overline{\mathrm{q}}$ multiplets of uncoloured gluons belonging to $\overline{8} \otimes \overline{8}$ and $8 \otimes 8$, plus Pati's and Salam's triplets of $X_{\mathrm{e}}$ and $X_{\mu}$ q l particles. Like Salam's 'prodigal' model, the model predicts (III) strong qq and (strongly reduced) 11 interactions, including $q \rightarrow \overline{1}+1+\bar{I}$ strong decays of free quarks. To these strong interactions, one must add, as a consequence of Cartan's triality principle (i.e. $8 \otimes \overline{8}=8^{\prime}, \overline{8} \otimes 8^{\prime}=8$ and $8 \otimes 8^{\prime}=\overline{8}$ ), strong interactions resulting from the two $\mathrm{SU}(3)$ triplets and singlets contained in $8^{\prime}$. In principle, evidently they strongly mix $F_{e}$-type and $F_{\mu}$-type fermions, but one sees that, if the corresponding masses are high enough, there is (Pati and Salam 1974a, b) no observable mixing of the $F_{e}$ and $F_{\mu}$ worlds, except through weak and electromagnetic interactions. One thus guarantees that normal hadrons (including $\mathrm{K}^{0}$ and $\overline{\mathrm{K}}^{0}$ ) may be considered predominantly as made up of e-quark type only and forbid transitions of the type $\mathrm{K}^{0} \rightarrow \mathrm{e}^{-}+\mu^{+}, \mathrm{e}^{+}+\mathrm{e}^{-}, \mu^{+}+\mu^{-}$. Finally, since all particles have non-zero bare mass, strong Lagrangians are invariant under $\mathrm{U}(1)$, which ensures parity conservation, i.e. $\mathrm{G}_{\mathrm{I}}$ (strong) $=\mathrm{U}(1) \otimes S U(3)$. As is well known, this implies the existence of a very light pseudoscalar boson, i.e. Weinberg's (1978) and Peccei and Quinn's (1977a, b) 'axion', which might have already been observed in anomalous redshifts recently discussed in the literature (Arp 1971, 1973, Pecker 1976). Moreover, the introduction of a random part in all gauge groups $G_{I}$ implies (Vigier 1962) that the corresponding Yang-Mills fields must have an effective non-zero mass, so that the model (as will be later discussed) implies the existence of the corresponding Higgs multiplets.

B. The model contains as a frame for weak interactions the maximal compact subgroup $U(1) \otimes U(1) \otimes S U_{L}(4)$, of which we identify the subgroup $U(1) \otimes S U_{L}(2)$ which preserves the 'weak' leptonic charge $\mathrm{H}_{3}$ with the weak-interaction gauge group of Weinberg and Salam, which appears, in terms of our internal motions, as the only and most probable candidate for a correct unification of weak and electromagnetic interactions. Indeed, with this assumption, one sees immediately that tables 1 and 2 now apparently agree with known facts of all types of strong-weak-electromagnetic interactions. Indeed, starting with $(\overline{8})_{\mathrm{L}}$, we can assume that it connects the usual SU(4) basic 4-representation of quarks $\mathrm{u}^{y}, \mathrm{~d}^{y}, \mathrm{~s}^{y}, \mathrm{c}^{\mathrm{y}}$ with the conjugate representation 4 of e-leptons in order to cancel the triangle anomalies. Since, independently of the $Q=f\left(H_{i}\right)$ definition, we must have a lepton EM charge sequence of the type $(-1,0,0,-1)$, the associated quark quartet must have the charges $(0,1,1,0)$, so that the model indeed implies the Nambu (1965) and Salam $(1974,1976)$ integer-charge assumption. Moreover, since isobasic spin and strangeness are now defined in the same way (III) for quarks and leptons, $\mu^{-}$and $\nu_{\mu}$ cannot be introduced in the same SU(4) quartet since they would generate strangeness, changing neutral currents and lepton number nonconservation. As a conseq rance, since experiment shows that $\tau$ can only be a sequential lepton (i.e. excited electron), we associate e-leptons with yellow quarks and $\mu$-leptons with blue quarks. The electric charge now exactly corresponds to $Q=H_{1}+H_{4}=$ $\left(\frac{1}{2}\right)\left(\lambda_{3}+\sqrt{\frac{1}{3}} \lambda_{8}-\sqrt{\frac{2}{3}} \lambda_{15}\right)+H_{4}$, where $\lambda_{i}$ denote the usual (Amati et al 1964) SU(4) generating matrices. Moreover, since the $H_{3}$-conserving Weinberg-Salam (WS) group is exactly embedded in $\mathrm{SU}_{\mathrm{L}}(4)$, as assumed recently by $Y$ ang $(1977,1978)$, we have only L-multiplets and quarks and leptons must have the same $\left(1-\mathrm{i} \gamma_{5}\right) / 2$ current parity. Weak CP-violation thus follows in this scheme from Okubo's suggestion (1968a, b) that 
weak Yang-Mills fields imply maximum parity violation, i.e. that we have $H_{\mathrm{w}}=$ $\mathrm{ig}\left(j_{\mathrm{w}}+1_{\mathrm{w}}\right) W_{\mu}+\mathrm{HC}$, with CP-parity -1 , the $W_{\mu}$ having strong interactions among themselves. It is also interesting to note that, in our model, any breaking of the Higg's scalars gauge symmetry leads to the Weinberg-Salam gauge theory, i.e. (1) SU(4) $\rightarrow$ $\mathrm{O}(5) \rightarrow \mathrm{SU}(2)$, the breaking of $\mathrm{SU}(2)$ with $\mathrm{U}(1)$ giving the WS theory; (2) SU(4) $\rightarrow$ $\mathrm{O}(4)=\mathrm{SU}(2) \otimes \mathrm{SU}(2) \rightarrow \mathrm{WS}$ with $\mathrm{U}(1)$ breaking; (3) $\mathrm{SU}(4) \rightarrow \mathrm{U}(1) \otimes \mathrm{SU}(2) \rightarrow \mathrm{SU}(2) \rightarrow$ WS.

Of course, all these decompositions imply, starting from the ws situation, the excitation of successive internal degrees of freedom so that the ws model is absolute for low-energy leptons, the Cabbibo angle appearing only among heavier particles, i.e. mixing of $d^{y}$ and $c^{y}$ (or $c^{b}$ and $u^{b}$ ), so that we recover Yang's result $(1977,1978$ ) for the Cabbibo angle.

C. The model is falsifiable in the sense that our internal motions in space-time imply the existence of only eight quarks $q$ and eight leptons 1 and yield the prediction $R=\sigma_{\mathrm{ee} \rightarrow \text { hadrons }} / \sigma_{\mathrm{e} \overline{\mathrm{e}} \rightarrow \mu \bar{\mu}}=\Sigma_{i} Q_{i}^{2}=6$, which can be compared with the observed value 5,5 at $\sqrt{s} \simeq 5 \mathrm{GeV}$ and represents a maximum possible value in this scheme. The model

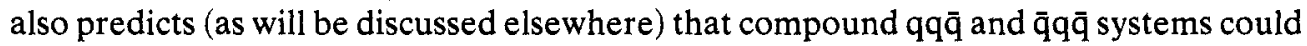
have a different lifetime, so that the faster decay of a fermionic compound antiparticle could explain why we live in a particle world, as suggested by recent astrophysical evidence (Demaret et al 1978).

\section{Acknowledgments}

The authors are grateful to Professors $M$ Flato and Sudarshan for criticism and suggestions in the early stages of this work. One of us (JPV) wants to thank Professor A Salam for hospitality and many discussions at the Trieste Center for Theoretical Physics, as well as the Institute of Physics, Belgrade, for facilities in the completion of this work. The authors also thank the French CNRS for support which made this joint research possible.

\section{References}

Amati D, Bacry H, Nuyts J and Prentki J 1964 Nuovo Cim. 341732

Arp H 1971 Science $\mathbf{1 7 4} 1189$

1973 IAU Symposium 58, Report Canberra

de Broglie L, Bohm D, Halbwachs F, Hillion P, Takabayasi T and Vigier J P 1963 Phys. Rev. 129 438, 451

Cartan E 1938 Leçons sur la théorie des spineurs (Paris: Hermann)

Chevalley C 1946 Theorie of Lie groups (Princeton: Princeton University Press)

Cufaro Petroni N and Vigier J P 1979 Lett. Nuovo Cim. 26149

Demaret J and Vandermeulen J 1978 Phys. Lett. 73B 471

Feynman R P, Kislinger M and Ravndal F 1971 Phys. Rev. D 32706

Flato M, Rideau G and Vigier J P 1965 Nucl. Phys. 61250

Gueret F, Merat P, Moles M and Vigier J P 1979 Lett. Math. Phys. 347

Gueret F, Vigier J P and Tait W 1973 Nuovo Cim. 17A 663

Halbwachs F and Souriau J M 1964 Preprint Faculté des Sciences de Marseille

Han M Y and Nambu Y 1965 Phys. Rev. 139B 1006

Katayama Y, Vigier J P and Yukawa H 1963 Prog. Theor. Phys. 29 468, 470

Kim Y S, Noz M E and Oh S H 1978 Univ. of Maryland CTP Tech. Rep. No 78-097 PP No 78-210

Nambu Y 1965 Proc. Second Coral Gables Conf. on Symmetry Principles at High Energy (San Francisco: Freeman) 
Okubo S 1968a Ann. Phys. 49219

1968b Nuovo Cim. 54A 491

1978 Phys. Rev. 183792

Pati J C and Salam A 1973 Phys. Rev. D 81240

- 1974a Preprint IC/74/7 Trieste

- 1974b Phys. Rev. D 10275

1975 Preprint University of Maryland GP 43662XX 1976 Preprint IC/76/63 Trieste

Pati J C, Salam A and Sakakibara S 1976 Preprint University of Maryland GP43662X

Peccei R D and Quinn H R 1977a Phys. Rev. Lett. 381440

1977b Phys. Rev. D 161791

Pecker J C 1976 Coll. Int. du CNRS 263451

Salam A 1968 in Elementary particle physics ed. N Svartholm (Stokholm: Svartholm)

- 1974a ICTP Preprint IC/74/87 Trieste 1976 Preprint IC/76/21

Takabayasi T 1965a Prog. Theor. Phys. 34124

- 1965b Phys. Rev. 138B 1381

1968 Prog. Theor. Phys. Suppl. 41130 1979 Prog. Theor. Phys. Suppl. 671

Vigier J P 1962 Nuovo Cim. 231171

1969 Lett. Nuovo Cim. 1445 1976 Lett. Nuovo Cim. 1541 1979 Lett. Nuovo Cim. 24265

Vigier J P and de Broglie L 1963 Compt. Rend. Acad. Sci. 2563551

Vigier J P and Gueret P 1970 Nuovo Cim. 67A 23

Weinberg S 1967 Phys. Rev. Lett. 191264

1978 Phys. Rev. Lett. 40223

Yang T C 1977 Phys. Lett. 70B 239

1978 Nucl. Phys. B 138345

Yukawa H 1950a Phys. Rev. 77219

— 1950b Phys. Rev. 801047 1953 Phys. Rev. 91 415, 416 1956 Prog. Theor. Phys. 16688 